\title{
Tumor ósseo multilobular em um cão
}

\author{
Multilobular Tumor of Bone in a Dog
}

\section{Aline de Biasi Bassani Gonçalves', Paulo Henrique Leal Bertolo ${ }^{2}$, Maria Eduarda Bastos Andrade Moutinho da Conceição ${ }^{2}$, Geórgia Modé Magalhães ${ }^{3}$, Marcio de Barros Bandarra ${ }^{4}$, Pamela Rodrigues Reina Moreira ${ }^{2}$ \& Rosemeri de Oliveira Vasconcelos ${ }^{2}$}

\begin{abstract}
Background: Multilobular tumor of bone (MTB) is a primary bone neoplasm, uncommon in dogs. This tumor was called of many names for a long time, as: chondroma rodens, multilobular osteochondrosarcoma, multilobular osteoma, multilobular chondroma, calcificating aponeurotic fibroma, although MTB was preferred chosen, because these other name could be correlated with humans' tumors. This tumor is observed specially in skull bone, although it was reported in zygomatic arc, hard palate, axilla, spine and penis. Mostly happen in big breeds dogs, and middle to old age patients. Clinical signs depending of region and how aggressive the tumor is, usually are related to compression of any structure. The growth of MTB is frequently slow and progressive, locally invasive, occurring relapse after surgical revomal, although the tumor has low to moderate metastatic potential. The aim of this study is to report a case of MTB in a female dog and describe anatomopathological changes.

Case: A female dog, mixed breed, 13 year-old, of middleweight was admitted in Pathology Department of College of Agricultural Sciences and Veterinary Medicine (FCAV-Unesp), Campus of Jaboticabal - SP, to be undergone to necropsy. The patient have never shown any epileptic crisis or neurologic signs. In macroscopic examination was found a mass in skull, which invaded the orbit and frontal sinus, but it was not invading brain cavity. The neoplasm had and irregular surface, firm consistent, color was white mixed to red areas, after cut it was granular and rough, and had some point mineralized areas. The lobs of lung had much firm masses, colored gray to white. In cytology it was observed fusiform to polyhedric isolated mesenchymal cells, moderated pleomorphic, basophilic cytoplasm, thin granulated nuclear chromatin, and visible nucleoli that was involved by eosinophilic extracellular matrix. The proposed diagnostic was bone sarcoma. Histopathological assessment showed mesenchymal neoplastic proliferation, and multilobular characteristic, the lobules had different sizes and was well organized, and they were separated by thin conjunctive septs. In the center of lobules, there was an "island" with mineralized or chondroitin bone matrix, in some of these islands there were osteoclast. The cells had moderated pleomorphism and low mitotic activity (three mitotic cells in ten high-power field). At least, it showed big necrosis areas and invasion of near tissue. In lung was observed metastatic areas, which had same histopathologic way of primary neoplasm in skull. That way, the histopathologic exam was similar to Multilobular tumor of bone grade II.

Discussion: The MTB is an uncommon neoplasm, which assaults mostly skull bone in large breeds dogs and middle age of eight years old. This case accord to literature about breed size, age and local of tumor. The clinical signs in this dog was related to region what it was growing and near structure compression. Besides that, the patient had lung metastasis, that is the principal metastatic sites according to literature. The Histopathologic exam showed the same characters of MTB, which was considered grade II according to literature. Despite MTB is an uncommon neoplasm in clinic of dogs and cats, the epidemiological knowns as age, breed and localization, anatomopathological changes, and histopathological exam allows to have a diagnosis, showing the importance of including this neoplasm in differential diagnosis of bone tumor in dogs.
\end{abstract}

Keywords: canine, skull, veterinary oncology, bone tumor.

Descritores: canino, crânio, oncologia veterinária, tumor ósseo. 


\section{INTRODUÇÃO}

O tumor ósseo multilobular (TOM) é uma neoplasia óssea primária, incomum na clínica de cães e sendo rara a ocorrência em outras espécies [4]. Por muito tempo foi descrito sob diferentes nomenclaturas, tais como, condroma rodens, osteocondrossarcoma multilobular, osteoma multilobular, condroma multilobular, fibroma aponeurótico calcificante, entre outros, porém o termo TOM é preferido, pois evita a correlação com termos aplicados a tumores similares em humanos e reconhece o fato dessa neoplasia apresentar comportamento benigno ou maligno [12].

Este tumor acomete principalmente os ossos do crânio, como a região frontal, parietal, occipital, mandíbula e maxila [4]. No entanto, esta neoplasia já foi relatada em outros sítios como arco zigomático [6], palato duro [1], axila [5], vértebras [10] e osso peniano [14].

Nos cães o TOM ocorre mais frequentemente em animais de meia idade a idosos, com média de idade de 8 anos, de raças médias a grandes e peso corporal de aproximadamente $29 \mathrm{~kg}$ [4,11], mas também já foi descrito em raças menores como o pequinês [8].

O comportamento biológico dessa neoplasia constitui geralmente um crescimento progressivo e lento, localmente invasivo, que pode comprimir estruturas adjacentes como o encéfalo, levar a recorrência pós-ressecção cirúrgica, porém com baixo a moderado potencial metastático $[4,7,11]$. Os sinais clínicos dependem da localização e da agressividade do tumor e

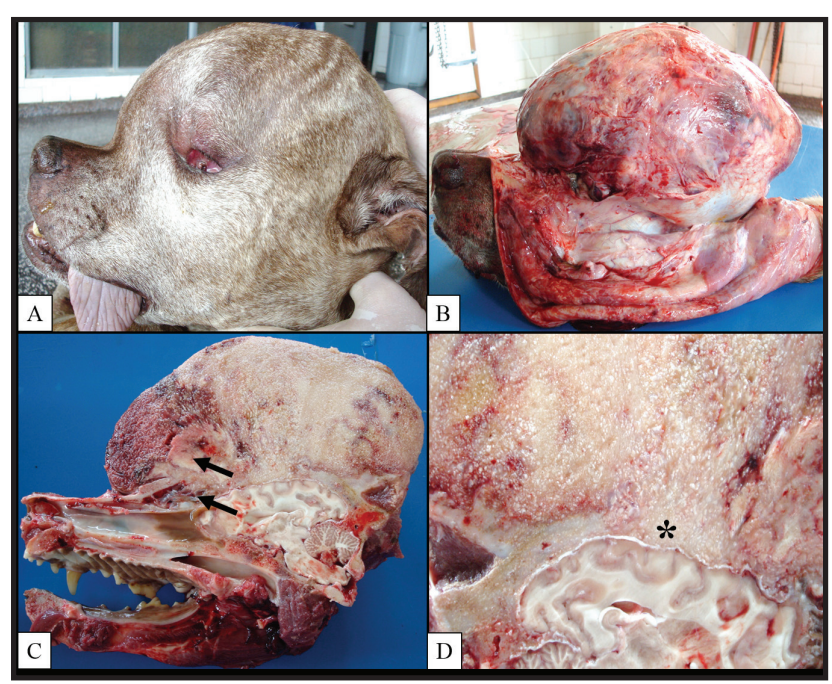

Figura 1. Canino com tumor ósseo multilobular. A- Canino apresentando aumento de volume simétrico na região frontal do crânio com deformidade facial. B- Aspecto macroscópico do crânio, após a remoção da pele. C- Crânio seccionado no plano sagital mediano apresentando massa brancacenta com áreas avermelhadas e comprometimento da órbita ocular e seio nasal (setas), sem invasão da caixa craniana. D- Notar detalhe da massa no limite da cavidade craniana $(*)$. geralmente estão relacionados à compressão de estruturas adjacentes [4].

O trabalho tem como objetivo relatar um caso de tumor ósseo multiblobular em uma cadela que apresentava aumento de volume simétrico em região frontal do crânio, descrevendo os achados anatomopatológicos.

\section{CASO}

Uma cadela, sem raça definida, de porte médio, com 13 anos de idade foi encaminhada ao Serviço de Diagnóstico Anatomopatológico do Departamento de Patologia Veterinária, da Faculdade de Ciências Agrárias e Veterinárias (FCAV-Unesp), Campus de Jaboticabal / SP, para a realização de exame necroscópico. O animal não apresentava histórico de episódios convulsivos ou qualquer alteração neurológica.

$\mathrm{Na}$ avaliação macroscópica constatou-se aumento de volume simétrico na região frontal do crânio e deformidade facial (Figura 1A e 1B), comprometendo a região ocular, devido a invasão bilateral da órbita. O crânio foi seccionado no plano sagital mediano e observou-se uma massa envolvendo os ossos frontal, parietal e occipital, que invadia o seio frontal (Figura 1C), mas não infiltrava na cavidade craniana, porém notou-se que a massa estava no limite de invadir esta cavidade. Na superfície de corte, a massa apresentou superfície irregular, consistência firme, coloração esbranquiçada mesclada com áreas avermelhadas, aparência granular grosseira e nódulos com áreas puntiformes de mineralização (Figura 1D). O pulmão apresentou vários nódulos, de tamanhos variados, brancoacinzentados, de superfície lisa, consistência firme, distribuídos de forma multifocal em todos os lobos pulmonares (Figura 2C).

Durante a necropsia foi realizada citologia pelo método de aspiração por agulha fina e os esfregaços obtidos foram corados utilizando kit de coloração Panótico Rápido $^{1}$ e também foram colhidos fragmentos da massa craniana e do pulmão para exame histopatológico. Os fragmentos dos tecidos foram fixados em solução de formol a $10 \%$, tamponado com fosfatos ( 0,15 Molar $)$ pH 7,2, durante $24 \mathrm{~h}$. O fragmento da massa craniana passou por processo de descalcificação em ácido nítrico $7,5 \%$, durante $48 \mathrm{~h}$. Posteriormente, os tecidos foram desidratados em soluções de concentração crescente de álcool, diafanizados em xilol e incluídos em parafina de acordo com a técnica histológica de rotina. Os blocos foram cortados na espessura de $5 \mu \mathrm{m}$ e as lâminas coradas com hematoxilina e eosina (HE) [13]. 


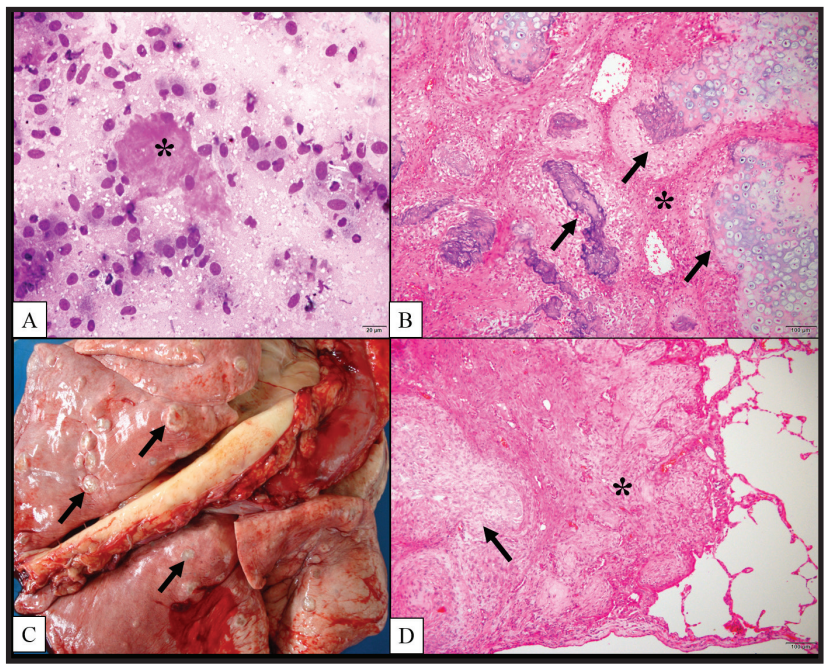

Figura 2. Canino com tumor ósseo multilobular. A- Citologia aspirativa mostrando células mesenquimais de aspecto alongado do núcleo, cromatina nuclear finamente granular, nucléolos visíveis e matriz extracelular eosinofílica em meio as células (*). [Panótico Rápido, Barra $=20 \mu \mathrm{m}$ ). B- Avaliação histopatológica mostrou aspecto multilobular com a formação de lóbulos de tamanhos variados de pequenos a grandes, bem organizados. Estes lóbulos eram delimitados por septos conjuntivos (*). Na região central dos lóbulos verificou-se "ilhas" contendo matriz óssea mineralizada ou condroide, ou ambas (setas). [H\&E, Barra $=100 \mu \mathrm{m}]$. C- Aspecto macroscópico do pulmão, mostrando vários nódulos, de tamanhos variados, esbranquiçados, de superfície lisa, consistência firme, distribuídos de forma multifocal em todos os lobos pulmonares (setas). D- Aspecto microscópico do pulmão com áreas de metástase $(*)$, que mantinham o padrão histopatológico da massa primária do crânio, como formação de lóbulos (seta). [H\&E, Barra $=100 \mu \mathrm{m}$ ].

$\mathrm{Na}$ análise citológica observou-se a presença de células mesenquimais fusiformes a poliédricas, dispostas de forma isolada, com pleomorfismo moderado, citoplasma basofílico, cromatina nuclear finamente granular, nucléolos visíveis, e também havia células envoltas por matriz extracelular eosinofílica (Figura 2A). Os achados citopatológicos foram sugestivos de um sarcoma ósseo.

Histologicamente observou-se aspecto multilobular com a formação de lóbulos de tamanhos variados de pequenos a grandes bem oragnizados. Estes lóbulos eram delimitados por septos conjuntivos delgados. $\mathrm{Na}$ região central dos lóbulos verificou-se "ilhas" contendo matriz óssea mineralizada ou condroide, ou ambas (Figura 2B) e em algumas áreas dessas "ilhas" observaram-se acúmulos de osteoclastos. As células apresentaram pleomorfismo moderado, a atividade mitótica era baixa (três figuras de mitose em 10 campos de grande aumento), observou-se também extensas áreas de necrose e comprometimento da margem. No tecido muscular adjacente à massa constatou-se atrofia e necrose de fibras musculares e discretos focos de infiltrado inflamatório mononuclear, composto predominantemente por linfócitos. No pulmão foi observado áreas de metástase, que mantinham o mesmo padrão histopatológico da massa primária do crânio (Figura 2D). Assim sendo, o padrão histopatológico do tumor foi compatível com tumor ósseo multilobular grau II, conforme proposto por Straw et al. [11].

\section{DISCUSSÃO}

O tumor ósseo multilobular é uma neoplasia incomum, que acomete principalmente os ossos do crânio em cães, com maior predisposição nos animais de raça de porte médio e grande, idade de surgimento entre 4 a 14 anos, com média de idade de 8 anos e não possui predisposição por raça e sexo [4]. No caso descrito, a cadela tinha 13 anos de idade, porte médio e a neoplasia ocorreu envolvendo alguns ossos cranianos, corroborando com a literatura.

No caso relatado, o tumor envolvia os ossos frontal, parietal e occipital, diferindo dos achados de outros autores que observaram a ocorrência do TOM somente na região occipital [3], apenas na região frontal [2], arco zigomático [6], palato duro [1], axila [5], vértebras [10] e osso peniano [14].

Os sinais clínicos apresentados pelos animais acometidos por esta neoplasia dependem da localização e da agressividade do tumor e geralmente estão relacionados à compressão de estruturas adjacentes [4]. Dentre os sinais clínicos apresentados pelo animal do presente relato observou-se o aumento de volume simétrico no crânio, a deformidade facial e a perda da visão, pela invasão tumoral na órbita. A dispneia possivelmente estava relacionada ao comprometimento dos seios nasais (invasão tumoral) e do pulmão pela presença de metástase. $\mathrm{O}$ animal não apresentou sinais neurológicos. Bohrer et al. [2] também relataram o aumento de volume craniano com deformidade facial e dificuldade visual em um cão com TOM, já Psychas et $a l$. [9] descreveram um caso de TOM em cão com invasão do crânio pela neoplasia e consequente compressão do encéfalo, resultando em quadro neurológico grave.

$\mathrm{O}$ animal do relato apresentou metástase pulmonar, que é o principal sítio metastático do TOM, segundo a literatura [11]. Resultados de diferentes estudos afirmam que cães podem desenvolver metástases após o tratamento e que o tempo médio para sua ocorrência varia entre $420-542$ dias $[4,11]$. O período de tempo para metástase reflete o comportamento biológico do TOM, de crescimento lento quando comparado a outros tumores ósseos malignos, como o osteossarcoma e condrossarcoma [12]. 
Os achados citopatológicos do caso em questão foram sugestivos de um sarcoma ósseo corroborando com os achados de outros estudos [2,9]. De acordo com a literatura, a citologia é uma ferramenta utilizada para auxiliar no diagnóstico dos sarcomas ósseos. Entretanto, amostras citológicas com a finalidade de identificar tumores ósseos específicos podem ser de difícil diagnóstico, pois os osteoblastos e condroblastos neoplásicos têm múltiplas características comuns, que impedem uma diferenciação precisa entre eles, tornando a histopatologia necessária para realizar um diagnóstico definitivo [12].

Os achados histopatológicos do presente caso evidenciaram um padrão característico de numerosos lóbulos, de células neoplásicas de origem óssea ou cartilaginosa, limitados por septos fibrosos delgados sendo conclusivo para o diagnóstico de TOM [12]. A graduação histológica do TOM, no presente estudo, foi classificada como grau II, com base nos critérios estabelecidos para espécie canina, como avaliação das margens, tamanho dos lóbulos, organização, número de mitoses, pleomorfismo celular e necrose como proposto por Straw et al. [11].

Embora o tumor ósseo multilobular seja considerado uma neoplasia incomum na clínica de animais de companhia, o conhecimento epidemiológico (idade, raça e localização), os achados anatomopatológicos, bem como os exames de citologia e histopatologia permitiram o diagnóstico definitivo, demonstrando a importância de incluir este tumor na lista de diagnósticos diferenciais de tumores ósseos em cães.

\section{MANUFACTURER}

${ }^{1}$ Labroclin Ltda. Pinhais, PR, Brazil.

Declaration of interest. The authors report no conflicts of interest. The authors alone are responsible for the content and writing of the paper.

\section{REFERENCES}

1 Banks T.A. \& Straw R.C. 2004. Multilobular osteochondrosarcoma of the hard palate in a dog. Australian Veterinary Journal. 82(7): 409-412.

2 Bohrer A.P.F., Santos F.R., Sperhacke C.J., Rasador F., Streck A.F., Jaconi F.C., Bastian N.C. \& França R.T. 2017. Tumor ósseo multilobular (Condroma rodens) em um cão. Acta Scientiae Veterinariae. 45(1): 231.

3 Costa T.S., Lopes N.L., Carvalho J.R.G., Mascarenhas M.B., Veiga C.C.P., Ramadinha R.R. \& Fernandes J.I. 2016. Tumor ósseo multilobular em cão - Relato de caso. Revista Brasileira de Medicina Veterinária. 36(2): 159-163.

4 Dernell W.S., Straw R.C., Cooper M.S., Powers B.E., La Rue S.M. \& Withrow S.M. 1998. Multilobular osteochondrosarcoma in 39 dogs: 1979-1993. Journal of American Animal Hospital Association. 34(1): 11-18.

5 Hay C.W., Roberts R. \& Latimer K. 1994. Multilobular tumour of bone at an unusual location in the axilla of a dog. Journal of Small Animal Practice. 35(12): 633-636.

6 Leonardi L., Carrano A., Stoppini L. \& Floris M. 2014. Multilobular tumor of the zygomatic bone in a dog. Open Veterinary Journal. 4(1): 9-11.

7 O'Brien M.G., Withrow S.J., Straw R.C., Powers B.E. \& Kipensteijn J.K. 1996. Total and partial orbitectomy for the treatment of periorbital tumors in 24 dogs and 6 cats: A retrospective study. Veterinary Surgery. 25(6): 471-479.

8 Pakhrin B., Bae I.H., Jee H., Kang M.S. \& Kim D.Y. 2006. Multilobular tumor of the mandible in a Pekingese dog. Journal of Veterinary Science. 7(3): 297-298.

9 Psychas V., Loukopoulos P., Polizopoulou Z.S. \& Sofianidis G. 2009. Multilobular tumour of the caudal cranium causing severe cerebral and cerebellar compression in a dog. Journal of Veterinary Science. 10(1): 81-83.

10 Stoll M.R., Roush J.K. \& Moisan P.G. 2001. Multilobular tumour of bone with no abnormalities on plain radiography in a dog. Journal of Small Animal Practice. 42(9): 453-455.

11 Straw R.C., LeCouteur R.A., Powers B.E. \& Withrow S.J. 1989. Multilobular osteochondrosarcoma of the canine skull: 16 cases (1978-1988). Journal of the American Veterinary Medical Association. 195(12): 1764-1769.

12 Thompson, K. G. \& Dittmer, K. E. 2017. Tumors of bone. In: Meuten D.J. (Ed). Tumors in domestic animals. 5th edn. Ames: John Wiley \& Sons Inc, pp.356-424.

13 Tolosa E.M.C., Rodrigues C.J., Behmer O.A. \& Freitas-Neto A.G. 2003. Manual de técnicas para histologia normal e patológica. 2.ed. São Paulo: Manole, 331p.

14 Webb J.A., Liptak J.M., Hewitt S.A. \& Vince A.R. 2009. Multilobular osteochondrosarcoma of the penis in a dog. Canadian Veterinary Journal. 50(1): 81-84.

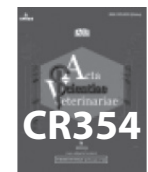

